\title{
Echolocating Distance by Moving and Stationary Listeners
}

\author{
Lawrence D. Rosenblum, \\ Michael S. Gordon, and Luis Jarquin \\ Department of Psychology \\ University of California-Riverside
}

\begin{abstract}
It has long been known that human listeners can echolocate a sound-reflecting surface as they walk toward it. There is also evidence that stationary listeners can determine the location, shape, and material of nearby surfaces from reflected sound. This research tested whether there is an advantage of listener movement for echolocating as has been found for localization of emitted sounds. Blindfolded participants were asked to echolocate a $3 \times 6 \mathrm{ft}$ wall while either moving or remaining stationary. After echolocating, the wall was removed, and participants were asked to walk to where the wall had been. Results showed that participants were somewhat more accurate with moving than stationary echolocation for some distances. A follow-up experiment confirmed that this moving advantage was not a function of a specific type of training or the multiple stationary positions available during moving echolocation. This subtle moving advantage might be a function of echoic time-to-arrival information.
\end{abstract}

Auditory information supports a blind individual's ability to navigate through the world. Information is provided by sound-emitting objects and events as well as from environmental surfaces that reflect sound. It has long been known that blind individuals can use reflected sound to avoid upcoming obstacles. This echolocation ability is similar to that performed by bats, dolphins, and birds (e.g., McFarland, 1987; Simmons, 1993). In a series of classic experiments, Cotzin, Dallenbach, and their colleagues (Cotzin \& Dallenbach, 1950; Supa, Cotzin, \& Dallenbach, 1944) demonstrated that both blind and (sighted) blindfolded human listeners can walk down a hallway and stop just before contacting a large board. These researchers determined that this ability to control approach was based on the reflected sound made

Requests for reprints should be sent to Lawrence D. Rosenblum, Department of Psychology, University of California-Riverside, Riverside, CA 92521.E-mail: rosenblu@citrus.ucr.edu 
available by the listeners' footsteps rebounding off of the board. In addition to obstacle approach, blind and blindfolded listeners have been shown to accurately judge the distance, shape, and texture of distal objects using reflected sound (e.g., Kellogg, 1962; Rice, 1967). In one particularly impressive example, a blind individual has been shown to use echolocation to successfully navigate a bicycle around the poles of an outdoor basketball court (Armstrong, 1996). In general, it is thought that the blind regularly use echolocation to avoid obstacles and perform other controlled encounters. It has also been suggested that sighted individuals make use of reflected sound (e.g., Stoffregen \& Pittenger, 1995). This is supported by findings that with relatively little training, blindfolded individuals can perform accurate echolocation (e.g., Cotzin \& Dallenbach, 1950; Kellogg, 1962; Rice, 1967).

Recently, an article in Ecological Psychology discussed human echolocation in the context of an ecological approach. Stoffregen and Pittenger (1995) posed echolocation as a prime example of a human perception-action ability, in that the listener actively induces the relevant acoustic structure. Based on this and other considerations, Stoffregen and Pittenger concluded that important concepts of the ecological approach can be illuminated by an action-based study of human echolocation. The experiments discussed here provide one of the first attempts to study the phenomenon of human echolocation from an ecological perspective. The experiments test echolocation using an active locomotory task and pose the question of whether the transforming reflective structure available to moving listeners facilitates distance judgment accuracy over that of stationary listeners. This question was anticipated in the article by Stoffregen and Pittenger (1995, pp. 186, 209).

Observer movement is known to enhance visual perception (e.g., Cutting, 1986; Rogers \& Collett, 1989). Informational dimensions such as motion parallax and time to arrival (TTA) have been shown to improve visual accuracy judgments of distance and shape (e.g., Srinivasan, 1992; Wallach \& O'Leary, 1979). Regarding audition, there is evidence that observer movement can enhance localization accuracy of emitted sounds. For sounds in the horizontal plane, head movements facilitate localization (Pollack \& Rose, 1967; Thurlow \& Runge, 1967). For auditory distance perception, there is conflicting evidence regarding the utility of movement. Simpson and Stanton (1973), Litovsky and Clifton (1992), and Rosenblum, Wuestefeld, and Anderson (1996) found no significant improvement in distance judgment accuracy with head movements. However, Ashmead, Davis, and Northington (1995; see also Speigle \& Loomis, 1993) found that with more extreme observer movements (5-10$\mathrm{ft}$ position changes), distance judgment accuracy can be enhanced.

Turning to echolocation, findings show that echolocation can be performed by both the locomoting listeners (Cotzin \& Dallenbach, 1950; Supa et al., 1944) and (relatively) stationary listeners (Kellogg, 1962; Rice, 1967). There is also evidence that porpoises use large head movements to echolocate targets in turbid water (e.g., Kellogg, 1962). As yet, however, no formal research has been conducted on whether listener movement enhances human echolocation. We now turn to the 
acoustical dimensions available during stationary and moving echolocation to catalog the potential information available in both contexts.

\section{POTENTIAL INFORMATION FOR STATIONARY AND MOVING ECHOLOCATORS}

For stationary echolocators, distance information could be available in the relation between emitted and reflected sound. Stoffregen and Pittenger (1995), among others (e.g., Bassett \& Eastmond, 1964; Clark, Pick, \& Wilson, 1975; Schenkman, 1985), suggested that the intensity, spectral, and temporal properties of the pulseto-echoed sound could be informative about surface distance. Distance changes these acoustic dimensions because as sound travels through air it loses overall energy, loses energy selectively across the frequency spectrum, and travels at a finite speed (e.g., Coleman, 1963). Thus, after a sound is emitted from a source, travels some distance, reflects off a surface, and travels back to an observer, its intensity, spectral structure, and arrival time are changed relative to the emitted sound. The utility of these dimensions will depend on the ear's sensitivity, potentially precluding their use at particularly short distances (Stoffregen \& Pittenger, 1995).

Under specific circumstances, another acoustic dimension might reveal echoic distance to stationary listeners. If the size of a reflecting surface is familiar, a listener could potentially use information about the extent of the acoustic solid angle of the surface as information about relative distance (Lee, 1990; Rosenblum, 1993). This acoustic solid angle is formed by the borders of the surface as the angle base, with the angle apex at the listener's ear. If the surface borders can be detected via relative echo delays, intensities, and spectral differences, this perceived extent could inform about relative distance. Again, for acoustic solid angle to be used by stationary listeners for judging distance, it is critical that the surface size is constant and known.

For moving echolocators, there are a number of additional acoustic dimensions available. For example, as a listener moves, there are systematic changes in the frequency dimension of the reflected sound as a result of the Doppler shift (e.g., Cotzin \& Dallenbach, 1950). An echoic Doppler shift occurs when a listener moves such that there is a compression (shortening) of wavelength for the waves between the listener and surface on approach and an expansion (lengthening) of wavelength for waves on retreat from the surface. These changes in wavelength can potentially alter the noticed pitch of the echoic sound. The Doppler shift has been found to be a useful dimension for locating moving sound sources (Jenison \& Lutfi, 1992; Lutfi \& Wang, 1999; Rosenblum, Carello, \& Pastore, 1987).

For echolocation, there is evidence that some species of bats make use of the Doppler shift and have extraordinary sensitivity to wavelength changes (Schnitzler \& Henson, 1980; Simmons, 1989). With regard to human echolocation, it is unclear whether the Doppler shift dimension is useful. Participants' introspective re- 
ports from early research suggested that Doppler-related information might be used. When asked which aspects of the changing sound they attended to, the individuals in Cotzin and Dallenbach's (1950) experiment reported noticing a pitch change in the reflected sound. However, follow-up experiments revealed that listeners were not as proficient at echo-based approach when the emitted source was a pure tone as when it was white noise. This finding is surprising in that although both types of sounds can be modified by the Doppler shift, sensory psychoacoustic data would suggest that pitch change sensitivity would be greater for pure tones (e.g., Terhardt \& Grubert, 1987).

The pitch change reported by Cotzin and Dallenbach's (1950) participants might actually reflect listener sensitivity to the changing interference pattern between emitted and reflected sound. This sweep, or ripplenoise pitch, is the phenomenological pitch (or timbre) change heard when a signal is added to itself across a changing time delay (Schenkman, 1985). This interference induces a selective modification in the spectrum of a complex sound. Schenkman (1985) suggested that at distances less than $2 \mathrm{~m}$, echolocators could use this change in phenomenological pitch (or timbre) to hear an upcoming surface. (The reader can easily experience ripple noise pitch change by forcing air through the lower teeth while moving an opened hand toward the mouth.)

Recently, another movement-induced acoustic dimension has been considered to support echolocation. Lee, van der Weel, Hitchcock, Matejowsky, and Pettigrew (1992) showed that an echoic TTA variable might be used by bats in navigating relative to a sound-reflecting surface. Following research in anticipatory judgments of looming visual (Lee, 1976; Lee, Young, Reddish, Lough, \& Clayton, 1983; Savelsbergh, Whiting, \& Bootsma, 1991; Schiff, 1965; Schiff \& Detwiler, 1979) and sound-emitting objects (Rosenblum, Wuestefeld, \& Saldaña, 1993; Schiff \& Oldak, 1990; Shaw, McGowan, \& Turvey, 1991), Lee and his colleagues showed how the time derivatives of changing echoic dimensions could be used for controlling approach. This tau variable informs about the TTA of an upcoming reflecting surface. Lee et al. (1992) suggested that echoic tau could be contained in the systematic change in pulse-to-echo delay, relative echoic intensity, and change in acoustic solid angle formed by the ear and any two points on the sound-reflecting surface. (Note that changing acoustic solid angle information in the service of tau does not depend on the surface extent being known.) Lee et al. (1992) found evidence that bats base their braking behavior on some form of the echoic TTA variable.

Stoffregen and Pittenger (1995) suggested that the frequency dimension can also lend itself to a time-to-contact (TTC) variable. Potentially, this systematic change in frequency could apply to either Doppler shift or spectral change frequency dimensions. In principle, the ripple noise pitch dimension might also change in a way to specify TTC, although formal modeling has not been done on this dimension. Other echolocation researchers have remarked on the potential informativeness of the time delay, intensity, and frequency dimensions as trans- 
formations induced by observer movement (e.g., Bassett \& Eastmond, 1964; Schenkman, 1985).

From the preceding analysis, it would seem that a moving echolocator would have available more acoustic dimensions specific to distance. This could provide moving echolocators with more robust information for distance, thereby facilitating accuracy. However, previous research has shown that successful echolocation can be performed by stationary listeners (Kellogg, 1962; Rice, 1967). In fact, Rice (1967) informally observed no advantage for head movements for his echolocators. At the same time, Kellogg (1962) noted that his blind participants were more apt to use exploratory head movements than sighted participants, possibly accounting for their superior performance. As yet, no formal comparison has been made between echolocation ability in moving versus stationary listeners. Furthermore, the listener movements that have been observed have been limited to head movements. It could be that more extreme point-of-observation movements would provide robust information and support greater accuracy. As discussed earlier, the extremity of movement has been shown to be a factor in distance judgment accuracy of sound sources (e.g., Ashmead et al., 1995; Speigle \& Loomis, 1993).

\section{THE OPEN-LOOP WALKING TASK}

In the following experiments, we implemented an action-based paradigm to examine whether listener movement facilitates echolocation of surface distance. Blindfolded listeners in the following experiments were asked to judge the distance to a large wall using an open-loop walking task (Ashmead et al., 1995; Loomis, Da Silva, Fujita, \& Fukusima, 1992; Rieser, Ashmead, Talor, \& Youngquist, 1990; Speigle \& Loomis, 1993; Thomson, 1983). Each trial involved having listeners (a) echolocate a wall while either remaining stationary or moving; (b) stand motionless with occluded ears as the wall was moved away; and (c) walk to the position where the wall had been during their echolocation. There is evidence that this task facilitates visual —and sound source—distance judgment accuracy (e.g., Ashmead et al., 1995; Rieser et al., 1990). Also, the open-loop walking task could allow for calibration of distance in action units (e.g., number of steps; Ashmead et al., 1995; Kim, Turvey, \& Carello, 1993).

Other steps were taken to facilitate listeners' optimal performance. Before the critical condition trials, participants were put through an extensive series of training trials. These trials involved walking all the way to the wall while continuously echolocating (e.g., Supa et al., 1944). On all of these training trials, verbal feedback was provided. Participants were also encouraged to explore different emitted sounds and use the ones they felt were most effective. Also, to potentially make the echoic structure more obvious, the target echolocating surface was large in both width and height (e.g., Schenkman \& Jansson, 1986). Finally, the experiments were conducted in a relatively quiet outdoor location with few other sound-reflecting surfaces nearby. 


\section{EXPERIMENT 1}

\section{Method}

Participants. Sixteen female students and 4 male students participated in this experiment for class credit. All participants were sighted and reported good hearing.

Materials. The experiment was conducted outdoors on a grassy field, approximately $15 \mathrm{~m} \times 40 \mathrm{~m}$. The use of an open environment was intended to reduce the amount of noncritical reverberant sound. The echolocated surface was a flat piece of drywall $(6 \mathrm{ft} 5 \mathrm{in} . \times 3 \mathrm{ft} \times 0.5 \mathrm{in}$., or $1.96 \mathrm{~m} \times 0.91 \mathrm{~m} \times 1.3 \mathrm{~cm})$. Participants were blindfolded using an opaque black plastic eye mask. To eliminate auditory information between trials, sound-attenuating headphones ( $21 \mathrm{~dB}$ reduction) were placed on participants' ears. None of the participants reported hearing between-trial information that might have facilitated their accuracy.

A guide string, stretched approximately $300 \mathrm{in.}(7.62 \mathrm{~m})$ between two wooden poles, was used to guide participants along the path toward the wall. Plastic stakes were set in the ground at 36, 72, 108, and $144 \mathrm{in} .(91.44 \mathrm{~cm}, 182.88 \mathrm{~cm}, 274.32 \mathrm{~cm}$, and $365.76 \mathrm{~cm}$; Distances $1-4$, respectively) adjacent to the string to delineate the wall distances for the experimenter (see Figure 1). Pilot experiments revealed that this range of distances was optimal for echolocated distance discrimination using an open-loop walking response. Although these wall distances are substantially shorter than those used in the sound source distance experiment of Ashmead et al. (1995), they intersect the range of distances used in the Speigle and Loomis (1993) experiment (discussed later).

Procedure. A $4 \times 2$ within-subjects factorial design was used for this experiment. For both training and critical trials, one experimenter issued the commands and another experimenter silently manipulated the wall. All instructions and

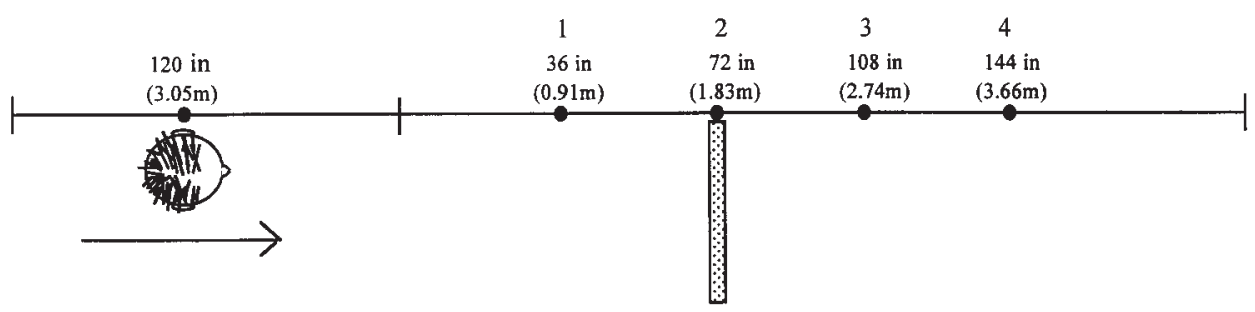

FIGURE 1 Schematic representation of the moving and stationary techniques for Experiment 1 with the wall positions marked. 
breaks were given in an area removed from the test site to prohibit visual access. Participants were instructed to avoid any visual contact with the test site.

The experiment began with a training session. For this part of the experiment, participants were led blindfolded into the test area by an experimenter. To familiarize themselves with the structure of the path, participants were briefly allowed to walk along the path using the guide string, without the wall in place. Immediately following this, participants were deafened with the headphones and aligned at the starting point $(0 \mathrm{in}$., or $0 \mathrm{~cm})$ as the wall was randomly placed at one of the four distances. Throughout the experiment, the wall was set into position concurrent with participant alignment at the starting point.

Forty training trials served to familiarize the participants with the echolocation task. During the training, participants experienced the wall 10 times at each distance. The instructions were to repeatedly produce a sound while walking slowly toward the wall. The goal was to stop as close to the wall as possible without touching. Participants were allowed to softly hit the wall when the distance was overestimated.

The sound used by participants was determined through their own experimentation. After 20, trials participants were limited to using a single chosen sound (e.g., the words hello or check, mouth clicks, etc.). Feedback was given after each trial by asking participants either to touch the wall or to attempt to move closer. After completing the training, participants were brought to the instruction area for critical trial instructions. Although no formal performance criteria were used to assess participants' accuracy after training, all seemed to perform the training task with accuracy and confidence by the end of the 40 trials.

The second part of the experiment consisted of 40 critical trials: 5 moving and 5 stationary at each of the four distances. (Although participants were given feedback as to the exact location of the distances during the training, no participants reported using a memorized step-counting technique to enhance their accuracy during the critical judgments. Also, see the following data.) Moving or stationary and distance condition trials were randomized with the constraint that no moving (or stationary) trial or a particular distance could occur more than three consecutive times.

For the 20 moving trials, participants were asked to echolocate the wall while walking $10 \mathrm{ft}$ (in $5 \mathrm{sec}$ ) up to the starting point. (Participants were always allowed to walk the full $10 \mathrm{ft}$, but if the time elapsed was not $5 \mathrm{sec}$, they were corrected on the subsequent trial.) When participants reached the starting point, an experimenter told them to stop. The sound-attenuating earphones were then quickly placed over the participants' ears, the wall was removed to a neutral location, and the participants were instructed (by a tap on the shoulder) to walk to the position where the where wall had been during the echolocation. The distance participants walked, from the starting point to the farthest tip of a toe, was measured to the nearest half inch $(1.27 \mathrm{~cm})$.

For stationary trials, participants were aligned $5 \mathrm{ft}(1.524 \mathrm{~m})$ from the starting point (i.e., the midway position traversed during the moving trials). Participants 
were given $5 \mathrm{sec}$ to echolocate the wall from this position. At the end of that time the experimenter said, "stop," and immediately placed the headphones on the participants. They were then walked to the starting point as the wall was removed. Participants were then instructed (by a shoulder tap) to walk to the location where the where wall had been during the echolocation. The whole experiment lasted approximately $90 \mathrm{~min}$ for each participants. Participants were given no performance feedback during the critical trials and a short break after 20 trials.

\section{Results and Discussion}

Analyses were performed to assess the accuracy and consistency of participants' judgments of the four target distances $(36,72,108$, and 144 in., or 91.44, 182.88, 274.32 , and $365.76 \mathrm{~cm}$ ) using the two techniques (moving and stationary). Accuracy was measured using the raw distance traversed by participants for each trial and the constant error ([ $\{$ distance traversed/target distance $\}-1] \times 100$; see Ashmead et al., 1995), a percentage reflecting the deviation of a judged distance from the target distance (see Figures 2 and 3). Positive constant error scores indicate a tendency

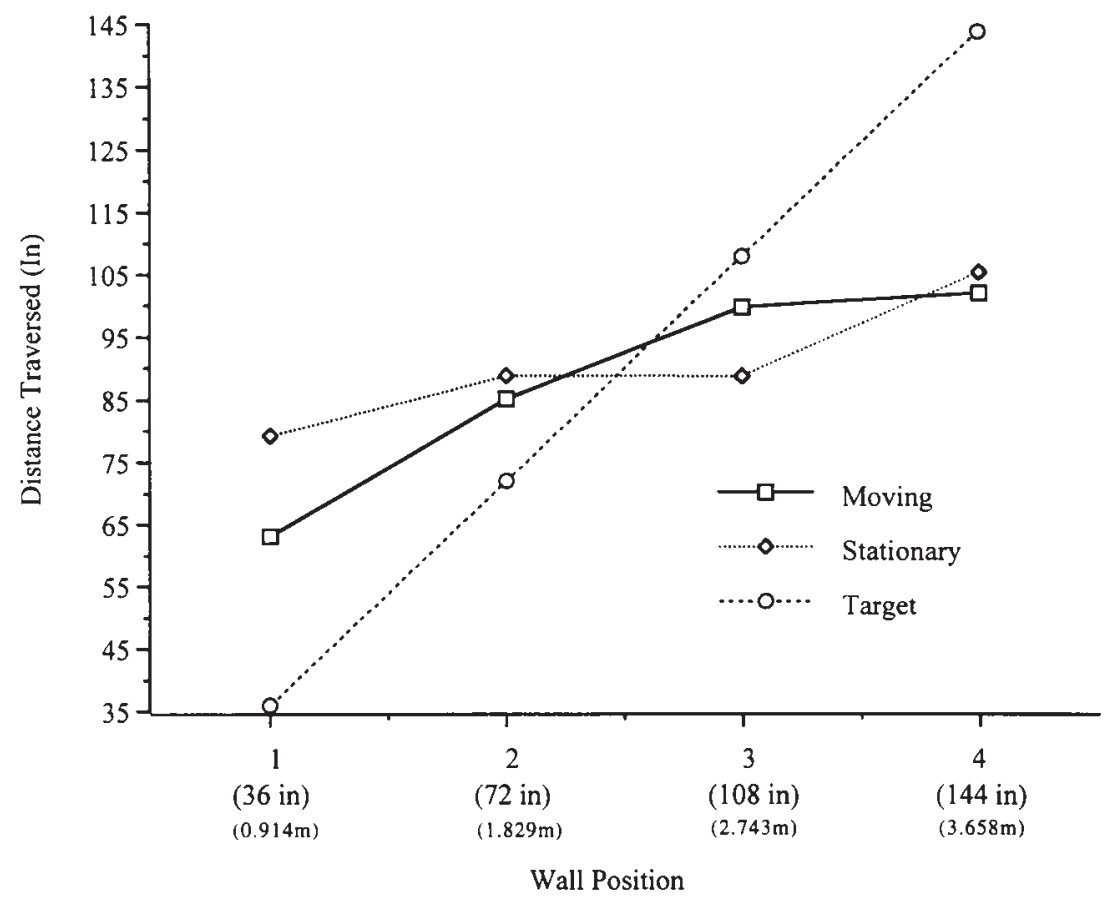

FIGURE 2 Mean distance traversed at each wall position as a function of technique in Experiment 1 . Target values represent ideal performance at each distance. 


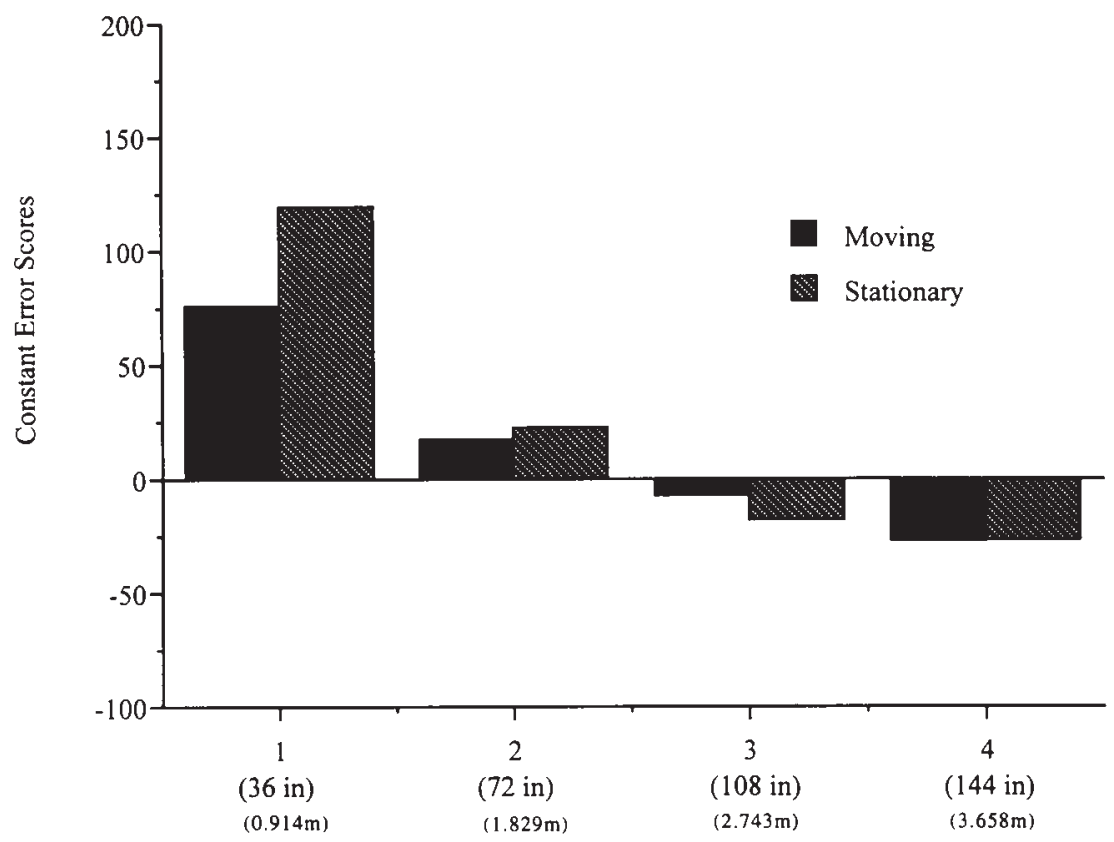

Wall Position

FIGURE 3 Mean constant error percentages at each wall position as a function of technique in Experiment 1.

to overshoot a target, and negative constant error scores indicate that participants are underestimating the target distance. Consistency was measured using standard deviations of the raw scores and standard deviation of the constant error scores (known as the variable error; Ashmead et al., 1995; see also Table 1).

An analysis of variance revealed a main effect of target distance for both the raw and constant error scores, $F(3,19)=15.867, p=.0001$, and $F(3,19)=76.271, p$ $<.0001$, respectively. These results indicate that judged distances did increase as the target distances increased. The constant error scores suggest that although participants could differentiate between the distances, judgments tended to be within a centralized, truncated range. This truncation is demonstrated in Figure 3 by the larger absolute values of the constant error scores at the extreme target distances (Distances 1 and 4). Constant error scores also marginally show a main effect of technique, $F(1,19)=4.209, p=.0542$, with moving judgments somewhat more accurate than stationary. The raw distances traversed, without regard to target distance, did not differ with technique, $F(1,19)=0.801, p=.3819$.

The raw scores did reveal a Technique $\times$ Target Distance interaction, $F(3,57)$ $=6.766, p=.0001$. Means comparisons revealed that moving judgments were significantly different from stationary judgments at target Distances 1 and $3, F(1,57)$ 
TABLE 1

Measures of Consistency: Standard Deviations and Variable Error Scores by Technique and Wall Distance for Experiment 1

\begin{tabular}{|c|c|c|c|c|}
\hline \multirow[b]{2}{*}{ Wall Distance } & \multicolumn{2}{|c|}{ Moving } & \multicolumn{2}{|c|}{ Stationary } \\
\hline & SD & Variable Error & $S D$ & Variable Error \\
\hline 1 & 23.35 & 59.22 & 29.76 & 76.72 \\
\hline 2 & 32.82 & 42.20 & 29.68 & 42.05 \\
\hline 3 & 23.49 & 22.61 & 26.81 & 24.67 \\
\hline 4 & 25.34 & 18.05 & 30.80 & 21.79 \\
\hline
\end{tabular}

Note. The standard deviations were derived from each participant's disparity around his or her own mean of raw distance traversed (inches).Variable error scores represent the deviation around the constant error scores, a participant's difference (\%) from ideal performance.

$=14.297, p=.0004$, and $F(1,57)=6.671, p=.0124$. In both comparisons, the moving judgments differed from the stationary by being closer to the targets. In addition, 15 of the 20 participants showed closer moving than stationary judgments at Distance 1 and 16 of the 20 participants showed a moving advantage at Distance 3. Discussion of why moving judgments might have been better at these distances are addressed in the general discussion. Moving and stationary trials also differed in their pattern of ordinality. Using the moving technique, Distances 1,2 , and 3 were ordinal: $1<2, F(1,57)=26.699, p<.0001 ; 2<3, F(1,57)=11.669, p=.0012$. Stationary judgments were ordinal for the target distances at the extremes: $1<2$, $F(1,57)=5.009, p=.0291 ; 3<4, F(1,57)=15.241, p=.0003$. No reliable differences were found in the analysis of standard deviations for the raw scores.

There was also a Technique $\times$ Distance interaction for the constant error scores, $F(3,57)=11.671, p<.0001$. Moving trials were more accurate than stationary at Distance $1, F(1,57)=39.552, p<.0001$.

The variable error scores had a main effect of distance, $F(3,19)=23.845, p=$ .0001 , indicating that as target distances increased, variable error decreased. No other variable error score comparison approached significance.

These data show that the manipulations did have some systematic influence over judgment accuracy, with less influence on judgment consistency. It is unclear why the manipulations would have relatively little effect on judgment consistency. One speculation is that the mean consistency values were rather high relative to the (truncated) mean distance values. This could indicate that within the truncated range of judged distances, the deviations were too high to allow for statistical differences to be observed. The remainder of this discussion is concentrated on the accuracy results.

Two main observations can be made from the accuracy findings. First, participants showed ordinal distance judgment accuracy using the open-loop walking task. Although the range of mean distance judgments was compressed relative to the ac- 
tual distances, an overall effect of distance was observed. Also, means tests showed significant ordinal differences among the three closest distances for the moving condition, and between the first and second, and third and fourth, distances for the stationary condition. It should be noted that this pattern of compressed range of mean distance judgments was replicated in many of the participants' individual data. This suggests that even though participants were given feedback during the training trials as to where the four critical distances were, it is unlikely that they used memorized step counts to enhance their absolute accuracy during the critical trials.

These accuracy results can be compared to previous findings on human echolocation. Kellogg (1962) used a method of constant stimuli to test distance discrimination in both sighted and blind individuals. He found that sighted individuals performed at near-chance levels at this task. However, the distances tested in Kellogg's experiment comprised a much smaller range $(1-3 \mathrm{ft})$ than those of our experiment. Also, unlike our experiment, Kellogg provided no formal training or feedback to his participants. This issue of training and perceptual learning might be important for the echolocating distance skill. In fact, Kellogg observed that blind participants fared much better at this task, approaching discrimination levels near that of visual distance judgments by sighted participants. It could be that if Kellogg's sighted participants were trained in a way similar to our participants, better echolocation performance would have been observed despite the extremely small range of distances.

Our results can also be compared to those from experiments on sound source distance using the open-loop walking task (i.e., Ashmead et al., 1995; Speigle $\&$ Loomis, 1993). First, as for our results, Speigle and Loomis found evidence of ordinal accuracy but less absolute distance accuracy. In fact, the results of Speigle and Loomis are similar to our echolocation findings in overall mean deviations from target distances and a compressed range of mean distance judgments relative to target distances. In the other experiment testing sound source distance judgments with the open-loop walking task, Ashmead et al. (1995) found both ordinal and impressive absolute accuracy. It is unclear why listeners in the Ashmead et al. experiment performed with more absolute accuracy than those in both the Speigle and Loomis experiment and our experiment. One distinction is that the target distances used in the Ashmead et al. study comprised a larger range (16.4-65.5 ft, or $5-20 \mathrm{~m})$, much of which was further than that used in the Speigle and Loomis $(6.6-19.7 \mathrm{ft}$, or 2-6 m) and current (3-12 ft, or 0.9-3.66 m) studies. Ashmead et al. also tested 15 target distances, whereas Speigle and Loomis tested three and our study tested four. Another methodological difference involved the walking judgments themselves. Listeners in Ashmead et al.'s experiments did not have to stop between hearing the source and walking to the position where the source had been. Both Speigle and Loomis's and our own methodologies required listeners to stop and don headphones to ensure that no spurious emitted sounds were heard. This break in the judgment task might have added to the absolute judgment error (Ashmead et al., 1995). Future research using open-loop walking judgments of 
source and echolocated distance judgments can be designed to examine which of these factors might have accounted for the difference in absolute accuracy.

The second main finding of our experiment was that echolocated distance accuracy was somewhat enhanced during moving versus stationary trials for some distances. Although the results show that this enhancement was subtle, they are the first to formally portray effects of listener movement on echolocation of distance. As stated earlier, informal observations have shown that blind echolocators tend to use head movements more often than sighted echolocators, which might account for their enhanced accuracy (Kellogg, 1962). Still, our findings are the first to suggest that movement can facilitate echolocation accuracy, to a small degree, in sighted individuals.

Before committing to this interpretation of our findings, however, alternative explanations must be considered. First, judgments in the moving condition might have been enhanced not because of the dynamic acoustic changes accorded movement but because listeners were allowed to echolocate from more than one stationary position (Ashmead et al., 1995). To test this possibility, a double-stationary condition was implemented in Experiment 2 wherein listeners echolocated at an initial stationary position, moved some distance, and then echolocated at a second position. This technique is borrowed from Ashmead et al. (1995), who addressed their observed moving advantage for sound source distance judgments in the same way.

A second reason performance might have been better in the moving condition is that our listeners went through substantial training in dynamic echolocation before the critical trials began. Recall that our training session involved listeners echolocating as they continually walked to the wall. This familiarity with dynamic echolocation might have later enhanced performance in the critical moving condition. To examine this possibility, the training session of Experiment 2 was redesigned so that listeners could gain experience with both moving and stationary echolocation skills. If the dynamic acoustic dimensions available to moving echolocators are more informative than those for stationary listeners, then accuracy (for some distances) should still be enhanced in the moving condition despite these methodological changes.

\section{EXPERIMENT 2}

\section{Method}

Participants. Twenty female students and 6 male students participated in this experiment for class credit. All participants were sighted and reported good hearing. None of them had participated in the previous experiment.

Materials. All the materials were the same as in Experiment 1 except that minor modifications were made to the echolocated surface. In Experiment 2, the 
wall consisted of a flat piece of drywall $(6 \mathrm{ft} \times 3 \mathrm{ft} \times 0.5$ in., or $1.83 \mathrm{~m} \times 0.914 \mathrm{~m} \times$ $1.27 \mathrm{~cm}$ ) with thin metal edges for reinforcement. Metal edges were used to avoid the decay that had affected the wall used in Experiment 1. A slightly shorter wall was used to maintain a weight consistent with the wall of Experiment 1 and alleviate concerns over additional noise as the wall was moved. Also a second guide string was added $2 \mathrm{in} .(5 \mathrm{~cm})$ below the original string for use during the stationary training trials (discussed later). This guide string was affixed with large plastic paper clips every $25 \mathrm{in} .(63.5 \mathrm{~cm})$ from the starting point to the farthest wall distance.

Procedure. As in Experiment 1, one experimenter issued all the instructions and the other manipulated the wall. Visual access to the test area was not permitted.

A set of 20 stationary training trials were added to alleviate concerns over unbalanced training in Experiment 1. During the training, moving and stationary trials and distances were randomly ordered with the constraints that condition (moving or stationary) or distance could not occur more than three consecutive times. For the stationary training, participants were asked to echolocate while standing still at each of the paper clips along the stationary guide string. After a brief echolocation, participants walked in total silence either to the next clip (to echolocate at that location) or as close to the wall as possible without touching it.

Training for the moving technique consisted of 20 trials identical to those described in the training for Experiment 1. Verbal or physical feedback was given after each trial as per Experiment 1 (for both moving and stationary conditions). Before each trial, the sound-attenuating headphones were placed on the participants, and they were aligned at the starting point. Concurrently, the wall was set at its next position.

The 40 critical trials consisted of 5 moving and 5 stationary trials at each of the four distances. Moving and stationary and distance trials were randomized with the constraint that no moving (or stationary) trial or a particular distance could occur more than three consecutive times.

The moving trials of Experiment 2 were nearly identical to the moving trials of Experiment 1. Participants were instructed to echolocate the wall while walking $10 \mathrm{ft}$ up to the starting point. For this experiment, however, participants were given $6 \mathrm{sec}$, rather than $5 \mathrm{sec}$, of echolocation, to correspond with the time needed for the stationary trials. After echolocation, the sound-attenuating earphones were quickly placed over the participants' ears, and the wall was removed to a neutral location. The participants were instructed (by a tap on the shoulder) to walk to the position where the wall had been during the echolocation.

The stationary trials consisted of echolocating at two positions (Ashmead et al., 1995): the back and front of the moving condition trajectory. Participants echolocated while stationary for $3 \mathrm{sec}$ positioned $10 \mathrm{ft}(3.05 \mathrm{~m})$ behind the starting point. Immediately after, the experimenter said, "stop," and placed the head- 
phones on the participants. They then walked to the starting point, where the headphones were removed, and were given an additional $3 \mathrm{sec}$ of echolocation. Following the second echolocation, the headphones were replaced on the participants, the wall was removed, and the participants were instructed (by a shoulder tap) to walk to the location where the wall had been.

Participants were given no performance feedback during the critical trials and a short break after 20 trials. The experiment took approximately $100 \mathrm{~min}$ for each participant.

\section{Results and Discussion}

Analysis of the raw distances traversed revealed a similar pattern of results as in Experiment 1 (see Figure 4). Distance judgments did increase with target distance, $F(3,25)=31.112, p<.0001$. There was also a marginal main effect of technique, $F(2,25)=3.690, p=.0662$; target distances tended to be judged as farther for moving trials than stationary trials.

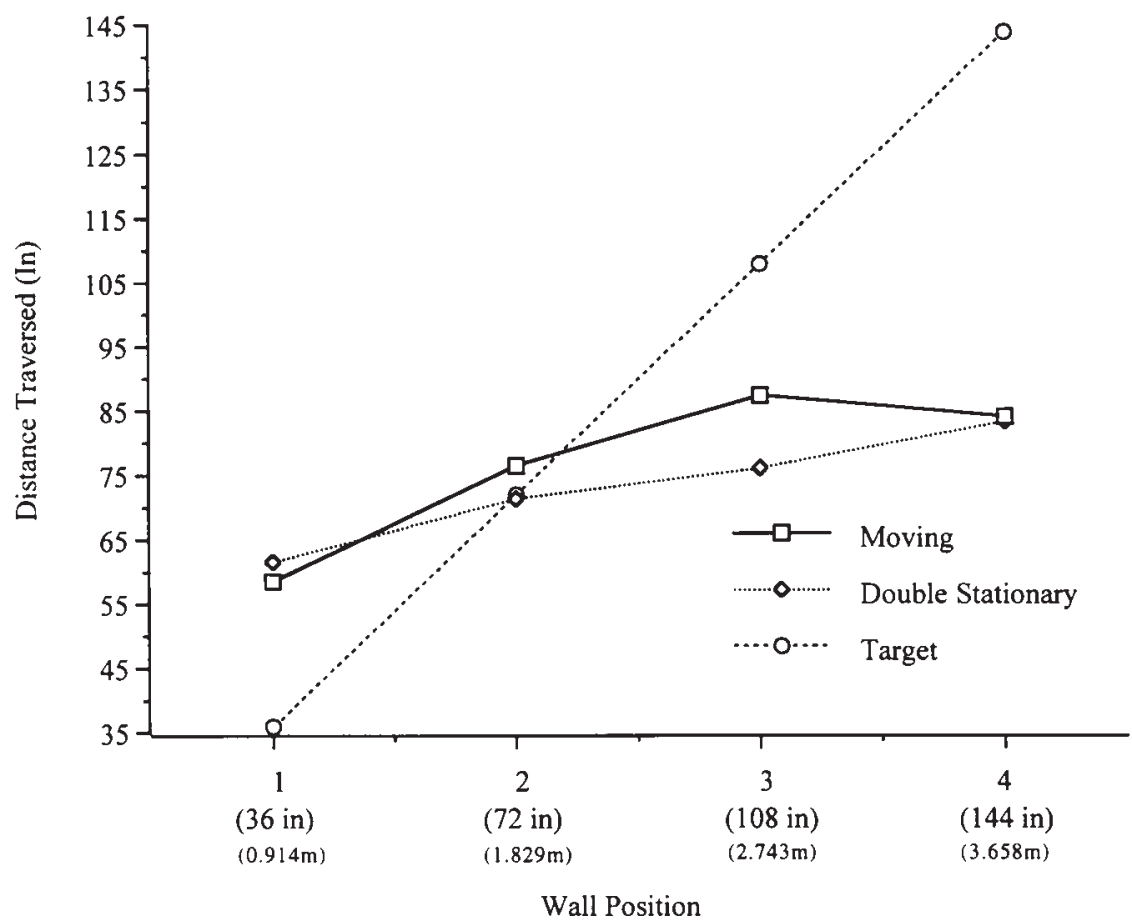

FIGURE 4 Mean distance traversed at each wall position as a function of technique in Experiment 2. Target values represent ideal performance at each distance. 
TABLE 2

Measures of Consistency: Standard Deviations and Variable Error Scores by Technique and Wall Distance for Experiment 2

\begin{tabular}{lccccc}
\hline & \multicolumn{2}{c}{ Moving } & & \multicolumn{2}{c}{ Double Stationary } \\
\cline { 2 - 3 } \cline { 5 - 6 } Wall Distance & SD & Variable Error & & SD & Variable Error \\
\hline 1 & 19.51 & 54.03 & & 18.56 & 51.19 \\
2 & 20.43 & 29.37 & & 17.71 & 24.57 \\
3 & 19.91 & 18.39 & & 21.03 & 19.89 \\
4 & 21.59 & 15.56 & & 21.65 & 14.07 \\
\hline
\end{tabular}

Note. The standard deviations were derived from each participant's disparity around his or her own mean of raw distance traversed (inches). Variable error scores represent the deviation around the constant error scores, a participant's difference (\%) from ideal performance.

There was a Distance $\times$ Technique interaction for the raw scores, $F(3,75)=$ $2.784, p=.0466$. Moving judgments were reliably different and closer to the target than stationary judgments at Distance $3, F(1,75)=9.373, p=.0031$. In addition, 19 of the 26 participants showed more accurate moving than stationary judgments at Distance 3. As in Experiment 1, moving trials also showed a different pattern of ordinality than double-stationary trials. Moving Distances 1,2 , and 3 were in order; $1<2, F(1,75)=23.703$, $p<.0001 ; 2<3, F(1,75)=8.704, p=.0042$. Stationary distances were ordinal at the extremes: Distance $1<2, F(1,75)=7.004, p$ $=.0099$, and Distance 3 marginally $<4, F(1,75)=3.787, p=.0554$. No reliable differences were found in the analysis of standard deviations (see Table 2).

Constant error was found to decrease from positive to negative values as distance increased, $F(3,25)=104.824, p<.0001$ (see Figure 5). As in Experiment 1, this result reveals a tendency to overshoot the closest targets and undershoot the farthest targets. For the constant error scores, there was no main effect of technique, $F(1,25)=2.709, p=.1123$, or Technique $\times$ Distance interaction, $F(3,75)$ $=1.829, p=.1491$. As with the raw scores, simple means tests were conducted to clarify the distance-technique relation. Moving trials were significantly more accurate than stationary trials at Distance $3, F(1,75)=4.363, p=.0401$. No other paired comparisons were significant. Variable error scores showed a main effect of distance, $F(3,25)=29.494, p=.0001$. As distance increased, variable error scores decreased. No other effects were found.

Again, the manipulations had a more systematic influence over judgment accuracy than judgment consistency. The remainder of this discussion is concentrated on the accuracy results.

The overall pattern of results for Experiment 2 is similar to that of Experiment 1. As before, listeners showed that ordinal accuracy and absolute mean distance judgments were compressed in range relative to the correct distances. Also, listeners in Experiment 2 fared somewhat better under the moving than (double) stationary condition, 


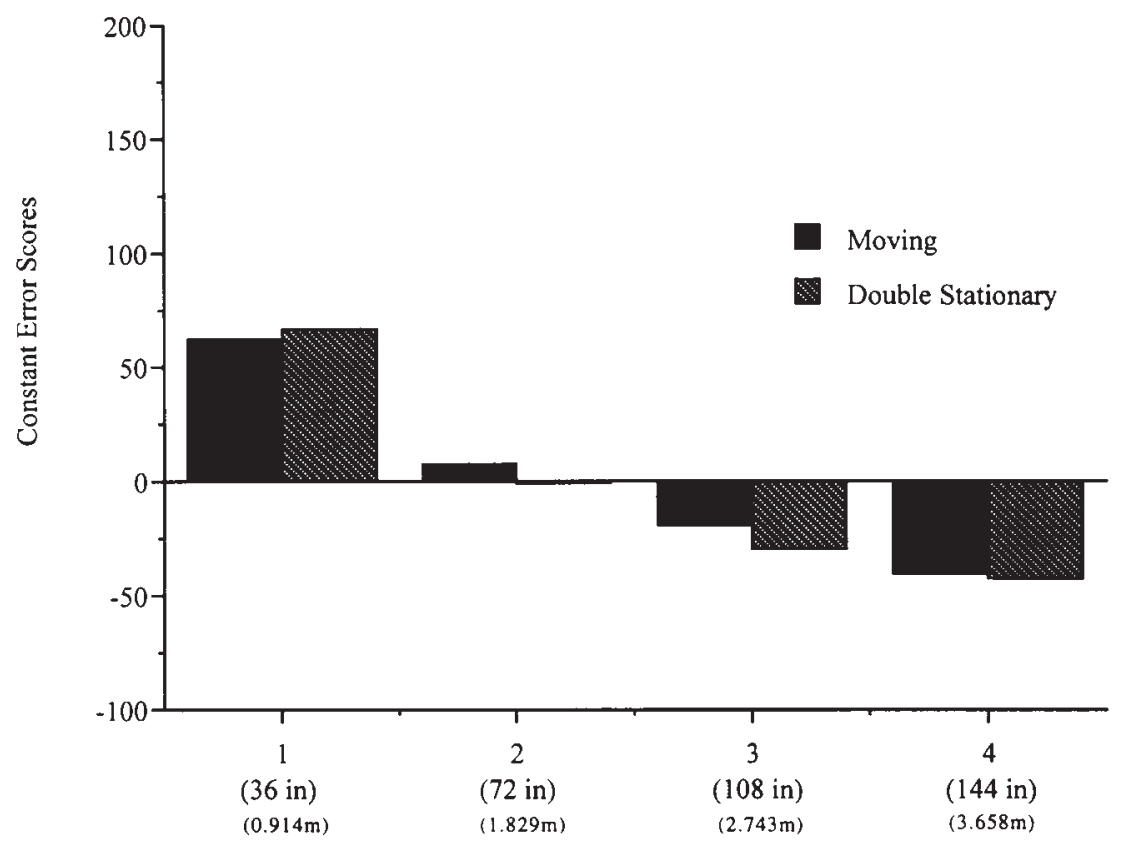

Wall Position

FIGURE 5 Mean constant error percentages at each wall position as a function of technique in Experiment 2.

with this advantage again dependent on the distance of the target surface. These results can be interpreted as evidence that (at least for Distance 3) the small advantage to the moving condition in Experiment 1 was a function of neither an unbalanced training session nor the fact that the moving condition contained multiple stationary positions. Potentially, the moving condition advantage for Distance 3 was a result of the availability of dynamic acoustic dimensions accorded a moving echolocator.

One difference between the results of our two experiments is that the moving condition advantage observed for Distance 1 in Experiment 1 disappeared in Experiment 2. It could be that either the added stationary training trials or multiple positions of the stationary condition accounted for this difference. However, because this difference was specific to a single distance, another explanation is implicated. It could be that the movement advantage for Distance 1 did not appear in Experiment 2 because both the moving and (double) stationary conditions of this experiment allowed listeners to echolocate at a position only $3 \mathrm{ft}(0.914 \mathrm{~m})$ from this distance. (Recall that the double-stationary condition involved echolocation at the farthest and closest positions of the trajectory.) It could be that at this close distance, stationary echolocation information is robust enough that listener movement is not enhancing (cf. Rice, 1967). Future research can examine this question. 


\section{GENERAL DISCUSSION}

The results of these experiments suggest that sighted listeners can distinguish the distance of an echolocated wall using an open-loop walking response. Our results also show that listener movement provides a small increase in echolocation accuracy for some distances. As stated, this is one of the first studies to formally show some advantage for listener movement in human echolocation. The advantage found for movement was maintained across both experiments for one surface distance (Distance 3). It is unclear why movement would have enhanced distance judgments of Distance 3 specifically. Potentially, the position of Distance $3(9 \mathrm{ft}$, or $2.74 \mathrm{~m}$ ) might have been such that the extra acoustic dimensions provided during moving echolocation were at their greatest relative salience. The static dimensions might have been robust enough to support the best possible performance at close distances (e.g., 1 and 2), whereas perception of the furthest distance (Distance 4:12 $\mathrm{ft}$, or $3.658 \mathrm{~m}$ ) was too far to be usefully enhanced by dynamic echoic acoustic dimensions.

Our relatively small effect of listener movement was more similar to the sound source distance findings of Speigle and Loomis (1993) than to those of Ashmead et al. (1995). Speigle and Loomis suggested that their methodology might have supported greater stationary accuracy because a constant source intensity was maintained throughout the experiment. With a constant source intensity, the subjective change in loudness across trials could be used by stationary listeners to judge source distance. Ashmead et al., on the other hand, varied source intensity (among three levels) from trial to trial, potentially forcing listeners to rely more on the dynamic acoustic dimensions when available.

This explanation has implications for our results. Although we exerted less control over the nature of our sound than either Speigle and Loomis (1993) or Ashmead et al. (1995), it was our sense that our participants attempted to produce emitted sounds with roughly the same intensity from trial to trial. In fact, there is evidence that blind individuals (Kish, 1995), as well as participants in previous echolocation experiments (e.g., Kellogg, 1962; Rice, 1967), tend to produce emitted sounds of constant intensity. Admittedly, no recordings or analyses of the participants' emitted sounds were conducted, rendering this claim as speculative. However, if this speculation holds true, consistency in the nature of the sound might have highlighted the relative direct to reflected sound intensity and spectral structure available to stationary listeners. The availability of this stationary echoic dimension could limit the enhancing utility of dynamic dimensions. This explanation would predict that if a less consistent emitted source were used (e.g., one that varied in intensity and timbre characteristics), then listeners, as in the Ashmead et al. study, might be forced to rely more on dynamic dimensions. Future research using either electronically or participant-varied emitted sounds could test this hypothesis.

The fact that our listeners displayed some success in both moving and stationary conditions suggests that both classes of acoustic dimensions were usable for 
echolocating distance. As stated, the stationary dimensions of pulse-to-echo intensity and spectral structure might have been enhanced by participants' attempts to maintain a consistent emitted sound. Temporal relations in the pulse-to-echo sound might have also supported listener performance during the stationary trials. Finally, in that the same reflective surface was used throughout the experiment, our listeners might have used acoustic solid angle information as projected size information for distance. Future research could involve manipulations of surface size across trials to determine the utility of acoustic solid angle information for stationary listeners.

On the other hand, the moving advantage observed for some of the distances might have been based on the availability of dynamic dimensions. As stated, these include specific changes in time, intensity, and spectral properties of the reflected sound. In addition, Doppler shift and ripple noise pitch might have enhanced distance judgments. More generally, the higher order changes in any of these dimensions (along with change in acoustic solid angle) might have provided auditory tau information, allowing listeners to more accurately gauge their walking judgments to Distance 3. (Informal postexperiment interviews suggested that participants had very little insight to the acoustic dimensions they used for this echolocation task.)

Throughout our discussion, we have been careful to refer to the echoic structure potentially supporting distance judgments as acoustical dimensions and not as information per se. From an ecological perspective, it is unlikely that any of these dimensions in their simplest form are truly informative in a specificational sense, that is, in a way that is fully supportive of action. For the reflected acoustic structure to be considered as true information, it must be described in action-relevant and, potentially, body-scaled, terms. It is likely that the acoustical specification of action-related echolocation is some higher order relation within or between these acoustic dimensions as they change with time. This was also suggested by Lee et al. (1992) in deriving acoustic tau as a constant time dimension in the form of intensity relation, echo delay, or changing acoustic angle. As suggested by Lee et al., this informational variable would be considered independent of the listener's velocity or the absolute magnitude of these acoustic dimensions.

\section{Evaluation of the Active Guidance Task}

As stated, our choice of open-loop walking task was motivated by considerations in the recent literature on sound source-and visual target—distance judgments (e.g., Ashmead et al., 1995; Bootsma, 1989; Rieser et al., 1990; Speigle \& Loomis, 1993). A number of studies have shown greater accuracy in the walking task relative to passive, extrinsic tasks such as verbal magnitude estimates. Another consideration identified in the recent literature is that the walking task could allow for calibration of distance in action-relevant units such as the number of steps (Ashmead et al., 1995; Kim et al., 1993). 
The outcome of our experiments suggests that the walking task was somewhat effective in evaluating echolocated distance accuracy. Although absolute accuracy revealed a compressed range of mean distance judgments relative to target distances, these mean scores show some consistent ordinality across techniques and experiments. Also, the patterning of distance judgments was comparable to the accuracy findings of Speigle and Loomis (1993) on sound source distance perception.

As stated, it is difficult to compare our results to the results on echolocating distance using other judgment paradigms. However, the ordinality displayed in our findings contrasts with the chance-level performance displayed by sighted individuals in the past research (e.g., Kellogg, 1962). In this sense, the walking task seemed to be an improvement toward facilitating optimal performance in echolocating distance.

Still, many of our participants commented on the difficulty of the critical (open-loop walking) trials relative to the training trials in which the surface was continually present during their approach. To ensure that our stationary condition only presented echoic structure to unmoving listeners, it was important to move the wall away during the walking judgment. However, it is likely that our training task was more similar to how echoic information is normally used by human listeners, that is, in relation to stable surfaces. In this sense, although our open-loop walking methodology might have captured many of the important dimensions of an action-related task, it might not be optimal as an example of echolocation in the "service of action" (Stoffregen \& Pittenger, 1995, p. 186). Stoffregen and Pittenger pointed out that the impressive echolocation performances observed with bats and dolphins have been in the context of more action-related tasks. These tasks involve guiding movement for the purposes of intercepting prey and navigating obstacles.

Our choices in methodology were constrained by our specific interest in whether dynamic acoustic dimensions enhance echolocation of distance. (We wanted to ensure that our listeners would be able to move over some substantial distance.) However, future research less concerned with the dynamic or stationary question can be designed to implement more "natural" action-oriented judgment tasks for human listeners. Examples of such tasks could include testing echolocated reachability of reflecting surfaces (Carello, Grosofsky, Reichel, Soloman, \& Turvey, 1989; Litovsky \& Clifton, 1992; Rosenblum et al., 1996), echolocated passability through apertures comprised of reflecting surface frames (Gordon \& Rosenblum, 1999; Warren \& Wang, 1987), echolocating the vertical extent of a surface to determine if it could allow stepping over (Pufall \& Dunbar, 1992; Schmuckler, 1996), or intercepting a looming surface through echolocation (e.g., Bootsma \& van Wieringen, 1990; Simmons, 1989).

However, the original methodology of having participants walk up to a wall and stop before contact (e.g., Cotzin \& Dallenbach, 1950; Supa et al., 1944) does seem to contain many of the important characteristics of an action-guided task. Listeners in this paradigm are able to induce specific acoustic changes from their own 
movement; that is, listeners are able to modulate and explore the acoustic information. The task of stopping before a wall also places listeners in a context of using the information in the service of action. Thus, the controlled walking methodology should continue to be of use in addressing action-related questions about human echolocation (discussed later).

\section{Questions for an Ecological Approach to Echolocation}

Although our experiments might be a useful first step toward establishing an action-motivated research program in human echolocation, many other important questions remain. For example, although we were able to show some advantage of listener movement, our research does not specifically address whether human listeners are sensitive to echoic tau information. As stated, Lee et al. (1992) showed that bats control their encounters as if guided by some form of echoic tau information. In these experiments, the approach velocity of the bat is measured relative to an upcoming aperture. Lee et al. (1992) showed that bats decelerate as if to keep the tau-dot value (the rate of change with respect to time) constant and between 0.5 and 1.0. Analogous studies could be easily conducted with human echolocators as they are asked to approach a stationary wall. The tau hypothesis would predict that a similar form of deceleration would be observed for human echolocators.

Another open issue for an event-based approach to echolocation concerns the nature of the emitted sound. Much of the extant echolocation research has been guided by a sonar metaphor in which listeners are thought to perform comparisons between self-emitted and reflected sound (e.g., Simmons, 1989; Stoffregen \& Pittenger, 1995). In fact, Stoffregen and Pittenger defined echolocation as using reflections of the self-generated sounds of the listener. In the engineering literature, sonar from receiver-generated signals is known as monostatic. However, echolocation as monostatic sonar is just one form of perception via reflected sound. Reflections of sounds emitted externally to the listener (in engineering, bistatic sonar) might be equally relevant to human listeners. Stoffregen and Pittenger did briefly discuss this issue, and they cited the minimal relevant research. They concluded that although such "third-party" (bistatic) pulse-to-echo information may be available, it is likely less useful than self-generated pulses. They argued that self-generated (monostatic) sounds afford signal modification and thus a more controlled form of perceptual exploration. In fact, bats are known for sophisticated control of their emitted sounds for exactly these purposes (e.g., Schnitzler \& Henson, 1980).

At the same time, Stoffregen and Pittenger (1995) did cite evidence that for human listeners, third-party (bistatic) sounds can provide reflecting surface detection accuracy comparable to that based on reflections of self-generated (monostatic) sounds (Rice, 1967; Supa et al., 1944). They discussed evidence that simulated 
surfaces can be reliably perceived through acoustic structure that is not self-generated (Seki, Kaji, Ifukube, \& Tanaka, 1993). In fact, there are recent reports that bats, dolphins, and sea lions can perform bistatic echolocation (Simmons, 1999; Turl, 1987; Xitco \& Roitblat, 1996). Furthermore, radars have been engineered that can decipher bistatic sonar for purposes of localization, surface mapping, and several other functions (Glaser, 1989; Lang \& Ermert, 1997). Whereas Stoffregen and Pittenger downplayed the evidence for bistatic echolocation in animals, we believe that this evidence invites a new conceptualization of echolocation.

It seems to us that the sonar metaphor of echolocation is limiting, especially as applied to human listeners. To get a sense of the space they occupy, human listeners likely make use of reflected structure initiated by signals generated from a myriad of sources (e.g., crowd noise, street sounds, ventilation systems). Perhaps a better metaphor for echolocation information would be the ambient optic array (Gibson, 1979/1986). In both cases, sources (e.g., the sun, a human voice) initiate an array of energy that envelops the observer and is lawfully structured by surrounding environmental surfaces. It is true that for most terrestrial environments, optic arrays are more durable and persistent than acoustic arrays. Thus, it might benefit the listener to have some control over the sound source in a way similar to how a spelunker benefits from having control over a flashlight as he or she explores a dark cave. However, for both the echolocator and spelunker, the critical information lies with the energy structured by reflective surfaces, not in comparisons between emitted and reflected energy. Just as the spelunker can make use of reflected structure initiated from another light source (a friend's flashlight), the echolocator should be able to make use of reflected structure that has not been self-generated (cf. Rice, 1967). We do not wish to downplay the importance of pulse-to-echo delay, intensity, and frequency shift information known to be useful for bats (e.g., Simmons, 1993). However, we believe that conceptualizing echolocation not as a sonar-type system but as based on a lawfully structured ambient acoustic array is more in line with an action-based approach.

With regard to human listeners, there is a growing literature on indirect sensitivity to reflected structure in the context of the precedence effect (for reviews see Blauert, 1997; Clifton \& Freyman, 1997; Hartman, 1996). The precedence effect refers to the ability to determine the location of a sound source by attending to its direct emissions, effectively suppressing signals that first reflect off of surfaces and reach the ears with some delay. The literature suggests that human listeners consider this echoic structure, and the architectural space it specifies, when localizing sources. This literature, along with the aforementioned research on bats, dolphins, and sea lions, as well as the work in engineering, warrants further consideration of reflected structure perception in terms of something other than a strict (monostatic) sonar metaphor.

Another issue for an action-based program pertains to the advantages of expertise in human echolocation. Testing sighted participants in echolocation experi- 
ments helps demonstrate the general availability and utility of echoic information (as well as issues in perceptual learning), but some aspects of human echolocation would be better examined through tests of more expert, visually impaired participants. Since the early human echolocation research (e.g., Kellogg, 1962), it has been known that blind echolocators show superior performance. This expert performance would more easily lend itself to evaluations of a number of the questions posed by an action account of echolocation. Expert performance has revealed important facts about action-related behavior in the context of visual TTA using birds (Lee \& Reddish, 1981), flies (Wagner, 1982), and human athletes (Warren, Young, \& Lee, 1986), as well as auditory TTA using bats (Lee et al., 1992) and visually impaired humans (Schiff \& Oldak, 1990). It is likely that the superior auditory attunement accorded visually impaired listeners (as well as their experience making everyday action-relevant judgments) would reveal important characteristics of human echolocation in an action-related context.

Whereas testing blind listeners might reveal important aspects of echolocation in and of itself, testing sighted listeners can address whether echoic information might normally be used to embellish vision. Stoffregen and Pittenger (1995), as well as others (Jenkins, 1985; Vicente, Christoffersen, \& Pereklita, 1995), suggested that reflected structure might be used to give observers a general sense of the dimensions of the environment they occupy, as well as information about their location in that environment. The research on the aforementioned precedence effect supports this suggestion. Research can be designed to examine the extent to which echoic and reflective acoustic structure might embellish exterospecific and propriospecific visual information. Accuracy, consistency, and reaction time of visual tasks might all be enhanced with the presence of reflected acoustic structure informative about observer location. Testing sighted echolocators could also allow examination of whether higher order information could exist in the relation between modality-specific dimensions as suggested by Stoffregen and Pittenger.

Sighted individuals are rarely conscious of their ability to use reflected sound as proprioceptive information. However, conscious awareness is not mandatory for successful echolocation. This fact was evidenced early on by erroneous selfreports of blind individuals that their ability to avoid collision was based on sensitivity to air pressure changes on the face (i.e., facial vision; Supa et al., 1944; see also Stoffregen \& Pittenger, 1995). In fact, this lack of conscious access to the sensory experience of echolocation might speak to the true perceptual nature (or automaticity) of the function. As with the other auditory functions of sound source localization (Konishi, 1991; Mattingly \& Liberman, 1990), speech perception (Fowler \& Rosenblum, 1991; Liberman \& Mattingly, 1985), and sound source identification (Gaver, 1993; Jenkins, 1985; Van Derveer, 1979; Warren $\&$ Verbrugge, 1984), perceptual awareness seems to be of distal object or event properties. An ecological perspective would expect this primacy of distal perceptual primitives and would expect that optimal performance would be observed in the context of action-related perceptual versus sensory judgments. 


\section{ACKNOWLEDGMENTS}

This research was supported by an intramural grant from the University of California. We gratefully acknowledge Fernando Cardenas, Jose Cervantes, Melina Doan, Veronica Felix, Todd Haidaczuk, Vy Lien, Veronica Ngo, and Bahareh Seifkabir for help in conducting these experiments. We also acknowledge the helpful comments of two anonymous reviewers.

\section{REFERENCES}

Armstrong, G. (Executive Producer). (1996). Sightings. Los Angeles: Ann Daniels Productions.

Ashmead, D. H., Davis, D. L., \& Northington, A. (1995). Contribution of listeners' approaching motion to auditory distance perception. Journal of Experimental Psychology: Human Perception and Performance, 21, 239-256.

Bassett, I. G., \& Eastmond, E. J. (1964). Echolocation: Measurement of pitch vs. distance for sounds reflected from a flat surface. Journal of the Acoustical Society of America, 36, 911-916.

Blauert, J. (1997). Spatial hearing: The psychophysics of human sound localization. Cambridge, MA: MIT Press.

Bootsma, R. J. (1989). Accuracy of perceptual processes subserving different perception-action systems. Quarterly Journal of Experimental Psychology, 41, 489-500.

Bootsma, R. J., \& Van Wieringen, P. C. (1990). Timing an attacking forehand drive in table tennis. Journal of Experimental Psychology: Human Perception and Performance, 16, 21-29.

Carello, C., Grosofsky, A., Reichel, F., Soloman, H. Y., \& Turvey, M. T. (1989). Visually perceiving what is reachable. Ecological Psychology, 1, 27-54.

Clark, N. V., Pick, G. F., \& Wilson, J. P. (1975). Obstacle detection with and without the aid of a directional noise generator. American Foundation for the Blind Research Bulletin, 29, 67-85.

Clifton, R. J., \& Freyman, R. L. (1997). The precedence effect: Beyond echo suppression. In R. Gilkey \& T. Anderson (Eds.), Binaural and spatial hearing in real and virtual environments (pp. 233-255). Mahwah, NJ: Lawrence Erlbaum Associates, Inc.

Coleman, P. D. (1963). An analysis of cues to auditory depth perception in free space. Psychological Bulletin, 60, 302-315.

Cotzin, M., \& Dallenbach, K. (1950). Facial vision: The role of pitch and loudness in the perception of obstacles by the blind. American Journal of Psychology, 63, 485-515.

Cutting, J. E. (1986). Perception with an eye for motion. Cambridge, MA: MIT Press/Bradford Books.

Fowler, C. A., \& Rosenblum, L. D. (1991). Perception of phonetic gesture. In I. G. Mattingly \& M. Studdert-Kennedy (Eds.), Modularity and the motor theory of speech perception (pp. 33-59). Hillsdale, NJ: Lawrence Erlbaum Associates, Inc.

Gaver, W. H. (1993). What in the world do we hear? An ecological approach to auditory event perception. Ecological Psychology, 5, 1-29.

Gibson, J. J. (1986). The ecological approach to visual perception. Hillsdale, NJ: Lawrence Erlbaum Associates, Inc. (Original work published 1979)

Glaser, J. I. (1989). Some results in the bistatic radar cross section (RCS) of complex objects. Proceedings of the IEEE, 77, 639-649.

Gordon, M. S., \& Rosenblum, L. D. (1999). Perception of acoustic occlusion using body-scaled judgments. Manuscript in preparation.

Hartman, W. M. (1996). Listening in a room and the precedence effect. In R. Gilkey \& T. Anderson (Eds.), Binaural and spatial hearing in real and virtual environments (pp. 256-279). Mahwah, NJ: Lawrence Erlbaum Associates, Inc. 
Jenison, R. L., \& Lutfi, R. A. (1992). Kinematic synthesis of auditory motion. Journal of the Acoustical Society of America, 92, 2458.

Jenkins, J. J. (1985). Acoustic information for objects, places, and events. In W. H. Warren \& R. Shaw (Eds.), Persistence and change: Proceedings of the first international conference on event perception (pp. 115-138). Hillsdale, NJ: Lawrence Erlbaum Associates, Inc.

Kellogg, W. N. (1962). Sonar system of the blind. Science, 137, 399-404.

Kim, N.-G., Turvey, M. T., \& Carello, C. (1993). Optical information about the severity of upcoming contacts. Journal of Experimental Psychology: Human Perception and Performance, 19, 179-193.

Kish, C. D. (1995). Evaluation of an echo-mobility program for young blind people. Unpublished master's thesis, California State University, San Bernadino.

Konishi, M. (1991). Neural mechanisms of binaural fusion. In I. G. Mattingly \& M. Studdert-Kennedy (Eds.), Modularity and the motor theory of speech perception (pp. 295-311). Hillsdale, NJ: Lawrence Erlbaum Associates, Inc.

Lang, M., \& Ermert, H. (1997). Linear synthetic aperture modes for ultrasonic pulse-echo imaging. Biomedizinische Technik, 42 (5), 108-115.

Lee, D. N. (1976). A theory of visual control of braking based on information about time-to-collision. Perception, 5, 437-459.

Lee, D. N. (1990). Getting around with light or sound. In R. Warren \& A. H. Wertheim (Eds.), Perception and control of self motion (pp. 487-505). Hillsdale, NJ: Lawrence Erlbaum Associates, Inc.

Lee, D. N., \& Reddish, P. E. (1981). Plummeting gannets: A paradigm of ecological optics. Nature, 293, 293-294.

Lee, D. N., Young, D. S., Reddish, P. E., Lough, S., \& Clayton, T. M. H. (1983). Visual timing in hitting an accelerating ball. Quarterly Journal of Experimental Psychology, 35, 333-346.

Lee, D. N., van der Weel, F. R., Hitchcock, T., Matejowsky, E., \& Pettigrew, J. D. (1992). Common principles of guidance by echolocation and vision. Journal of Comparative Psychology, 171, 563-571.

Liberman, A. M., \& Mattingly, I. G. (1985). The motor theory of speech perception revised. Cognition, $21,1-36$.

Litovsky, R. Y., \& Clifton, R. K. (1992). Use of sound-pressure level in auditory distance discrimination by 6-month-old infants and adults. Journal of the Acoustical Society of America, 92, 794-802.

Loomis, J. M., Da Silva, J. A., Fujita, N., \& Fukusima, S. S. (1992). Visual space perception and visually directed action. Journal of Experimental Psychology: Human Perception and Performance, 18, 906-921.

Lutfi, R. A., \& Wang S. (1999). Correlational analysis of acoustic cues for the discrimination of auditory motion. Journal of Acoustic Society of America.

Mattingly, I. G., \& Liberman, A. M. (1990). Speech and other auditory modules. In G. M. Edelman, W. E. Gall, \& W. M. Cowan (Eds.), Signal and sense: Local and global order in perceptual maps (pp. 501520). New York: Wiley.

McFarland, D. (1987). The Oxford companion to animal behavior. New York: Oxford University Press.

Pollack, I., \& Rose, M. (1967). Effect of head movement on localization of sounds in the equatorial plane. Perception $\mathcal{E}$ Psychophysics, 2, 591-596.

Pufall, P. B., \& Dunbar, C. (1992). Perceiving whether or not the world affords stepping onto and over: A developmental study. Ecological Psychology, 4, 17-38.

Rice, C. E. (1967). Human echo perception. Science, 155, 656-664.

Rieser, J. J., Ashmead, D. H., Talor, C. R., \& Youngquist, G. A. (1990). Visual perception and the guidance of locomotion without vision to previously seen targets. Perception, 19, 675-689.

Rogers, B. J., \& Collett, T. S. (1989). The appearance of surfaces specified by motion parallax and binocular disparity. Quarterly Journal of Experimental Psychology, 41, 697-717.

Rosenblum, L. D. (1993). Acoustic information for controlled collisions. In A. Schick (Ed.), Contributions to psychological acoustics (pp. 303-322). Oldenburg, Germany: Bibliotheks- und Informatiossytem der Carl von Ossietzky Universität Oldenburg. 
Rosenblum, L. D., Carello, C., \& Pastore, R. E. (1987). Relative effectiveness of three stimulus variables for locating a moving sound source. Perception, 16, 175-186.

Rosenblum, L. D., Wuestefeld, A. P., \& Anderson, K. L. (1996). Auditory reachability: An affordance approach to the perception of sound source distance. Ecological Psychology, 8, 1-24.

Rosenblum, L. D., Wuestefeld, A. P., \& Saldaña, H. M. (1993). Auditory looming perception: Influences on anticipatory judgments. Perception, 22, 1467-1482.

Savelsbergh, G. J., Whiting, H. T., \& Bootsma, R. J. (1991). Grasping tau. Journal of Experimental Psychology: Human Perception and Performance, 17, 315-322.

Schenkman, B. N. (1985). Human echolocation: A review of the literature and a theoretical analysis. Uppsala Psychological Reports, 379.

Schenkman, B. N., \& Jansson, G. (1986). The detection and localization of objects by the blind with the aid of long-cane tapping sounds. Human Factors, 28, 607-618.

Schiff, W. (1965). Perception of impending collision: A study of visually directed avoidant behavior. Psychological Monographs: General \& Applied, 79(11), 1-26.

Schiff, W., \& Detwiler, M. L. (1979). Information used in judging impending collision. Perception, 8, $647-658$.

Schiff, W., \& Oldak, R. (1990). Accuracy of judging time to arrival: Effects of modality, trajectory, and gender. Journal of Experimental Psychology: Human Perception and Performance, 16, 303-316.

Schmuckler, M. A. (1996). Development of visually guided locomotion: Barrier crossing by toddlers. Ecological Psychology, 8, 209-236.

Schnitzler, H. U., \& Henson, O. W. (1980). Performance of airborne animal sonar systems: I Microchiroptera. In R. G. Busnel \& J. F. Fish (Eds.), Animal sonar systems (pp. 109-181). New York: Plenum.

Seki, Y., Kaji, K., Ifukube, T., \& Tanaka, Y. (1993). On the relation between obstacle sense of the blind and variation of the soundfield. In Proceedings of the 19th Sensory Substitution Symposium, Tokyo (pp. 9-15). Tsukuba, Japan: Association of Sensory Substitution.

Shaw, B. K., McGowan, R. S., \& Turvey, M. T. (1991). An acoustic variable specifying time-to-contact. Ecological Psychology, 3, 253-261.

Simmons, J. A. (1989). A view of the world through the bat's ear: The formation of acoustic images in echolocation. Cognition, 33, 155-199.

Simmons, J. A. (1993). Evidence for perception of fine echo delay and phase by the FM bat, Eptesicus fuscus. Journal of Comparative Physiology: Sensory, Neural, and Behavioral Physiology, 172, 533-547.

Simmons, J. A. (1999, August). The role of auditory scenes and object flow in echolocation. Paper presented at the 10th International Conference on Perception and Action, Edinburgh, Scotland.

Simpson, W. E., \& Stanton, L. D. (1973). Head movement does not facilitate perception of the distance of a sound source. American Journal of Psychology, 86, 151-159.

Speigle, J. M., \& Loomis, J. M. (1993). Auditory distance perception by translating observers. In Proceedings of IEEE 1993 Symposium on Research Frontiers in Virtual Reality (pp. 92-99). San Jose, CA: Institute of Electrical and Electronics Engineers Computer Society.

Srinivasan, M. V. (1992). Distance perception in insects. Current Directions in Psychological Science, 1, $22-26$.

Stoffregen, T. A., \& Pittenger, J. B. (1995). Human echolation as a basic form of perception and action. Ecological Psychology, 7, 181-216.

Supa, M., Cotzin, M., \& Dallenbach, K. M. (1944). Facial vision: The perception of obstacles by the blind. American Journal of Psychology, 57, 133-183.

Terhardt, E., \& Grubert, A. (1987). Factors affecting pitch judgments as a function of spectral composition. Perception $\mathcal{E}$ Psychophysics, 42, 511-514.

Thomson, J. A. (1983). Is continuous visual monitoring necessary in visually guided locomotion? Journal of Experimental Psychology: Human Perception and Performance, 9, 427-443. 
Thurlow, W. R., \& Runge, P. S. (1967). Effect of induced head movements on localization of direction of sounds. Journal of the Acoustical Society of America, 42, 480-488.

Turl, C. W. (1987). The ability of the California sea lion, Zalophus californianus, to bistatically detect and localize echoes from underwater targets. Journal of the Acoustical Society of America, 82, 381-383.

Van Derveer, N. J. (1979, June). Acoustic information for event perception. Paper presented at E. J. Gibson Celebration, Cornell University, Ithaca, NY.

Vicente, K. J., Christoffersen, K., \& Pereklita, A. (1995). Supporting operator problem solving through ecological interface design. IEEE Transactions on Systems, Man, Ë Cybernetics, 25, 529-545.

Wagner, H. (1982). Flow-field variables trigger landing in flies. Nature, 297, 147-148.

Wallach, H., \& O'Leary, A. (1979). Adaptation in distance perception with head-movement parallax serving as the veridical cue. Perception \& Psychophysics, 25, 42-46.

Warren, W. H., \& Verbrugge, R. R. (1984). Auditory perception of breaking and bouncing events: A case study in ecological acoustics. Journal of Experimental Psychology: Human Perception and Performance, 10, 704-712.

Warren, W. H., Jr., \& Wang, S. (1987). Visual guidance of walking through apertures: Body-scaled information for affordances. Journal of Experimental Psychology: Human Perception and Performance, 13, 371-383.

Warren, W. H., Young, D. S., \& Lee, D. N. (1986). Visual control of step length during running over irregular terrain. Journal of Experimental Psychology: Human Perception and Performance, 12, 259-266.

Xitco, M. J., Jr., \& Roitblat, H. L. (1996). Object recognition through eavesdropping: Passive echolocation in bottlenose dolphins. Animal Learning and Behavior, 24, 355-365. 